\title{
On the reconstruction capability of the linear sampling method
}

\author{
Morteza Ghaderi Aram
}

Department of Electrical and Computer Engineering, K. N. Toosi University of Technology, Tehran, Iran

Abstract: Linear Sampling Method (LSM), although a simple and fast qualitative method, encounters some limitations and restrictions when it comes to measurement set-up and realistic implementation. Addressing some difficulties arising from somewhat complicated measurement structures used to gather raw data for the reconstruction algorithm, this communication tries to show the effect of mutual coupling between antennas utilized in the measurement chamber. Another tangible effect of the antenna arrangement, covered here through reconstructing a reference scenario of Fresnel Institute dataset, has to do with the aspect-limited nature of scan lines. Reconstructions based on both simulated and measured data are reported.

Keywords: inverse scattering; microwave imaging; qualitative methods; LSM

\section{Introduction}

The main purpose of microwave imaging (MWI) systems is to interrogate an investigation domain by means of microwaves and then convert the backscattered signals into interpretable images of the scenario. Because of their potential for giving a clear image of every discontinuity in constitutive parameters of the investigation domain, MWI methods have paved the way for their presence in different contexts from GPR and through-the-wall radar imaging to biomedical applications for breast cancer detections and cerebral monitoring, to name but a handful.

Needs for fast and reliable methods to convert scattered field distributions into interpretable images have been conducive to introduction of such qualitative methods as sampling methods (SM), level set, and so on ${ }^{[1-3]}$. Of all these methods, SMs which are themselves comprised of linear sampling method (LSM) and factorization method (FM) have been attracting increasingly attention since they tend to solve the scattering equations in a linear manner without using any approximations like Born approximation. This is why SMs are not only fast reconstruction methods, but they enjoy a wide range of applicability in real scenarios as well.

It is shown ${ }^{[4]}$ that LSM can be interpreted as a synthetic focusing problem thus sharing all the limitations and merits of these classic electromagnetic problems. In fact, for the measurement probes to be implemented, there are several considerations to take into account. The first one is related to the idea that mutual coupling between adjacent elements has a detrimental effect on the resultant image, so it must be avoided as much as possible. That the elements cannot always be arranged in a full circle all around the investigation domain is the second consideration which is often referred to as the aspect-limited feature of the problem.

The paper is organized as follows. Section II describes the forward problem (data gathering and validation) as well as the formulation of the inversion method. The reconstruction results under different circumstances and limitations are presented in section III. Finally, the last section concludes the paper

Copyright (C) 2018 Morteza Ghaderi Aram

doi: 10.18686/esta.v5i1.59

This is an open-access article distributed under the terms of the Creative Commons Attribution Unported License

(http://creativecommons.org/licenses/by-nc/4.0/), which permits unrestricted use, distribution, and reproduction in any medium, provided the original work is properly cited. 


\section{Description of the Problem}

Time convention throughout the paper is assumed to be $e^{\mathrm{i} \omega t}$ and suppressed.

\subsection{Simulated Raw Data}

Shown in Figure. 1 is a 2D scenario comprised of antennas in a circular scan line surrounding a cylindrical object, where the system is considered to be elongated along the z-axis. So the variation in this direction can be ignored and scattering equations can be turned into scalar ones. Raw data is indeed a matrix whose elements are the scattered electric field distributions collected in a multistatic/multiview configuration.

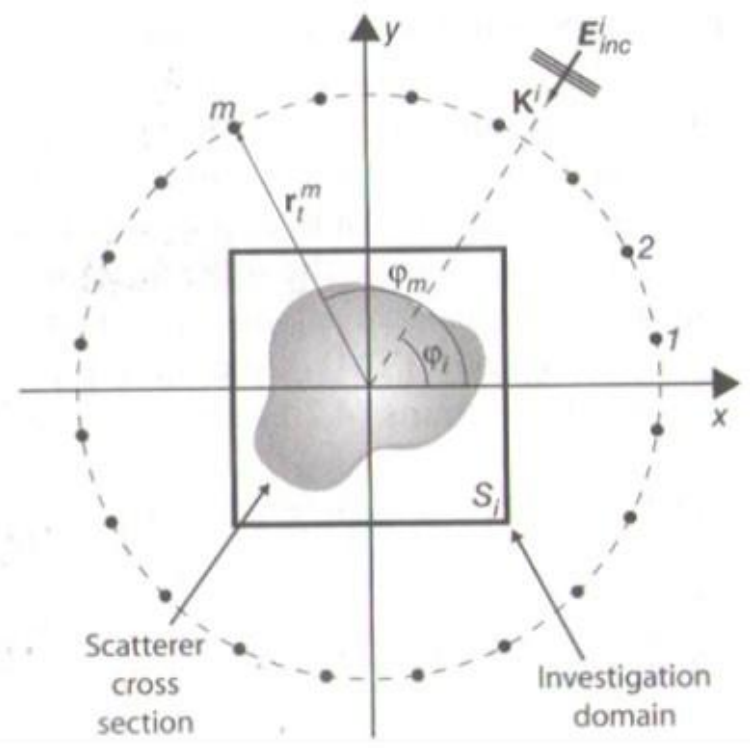

Fig. 1 The 2D scenario which depicts the antennas (located in a circular scan line in far-field) and the investigation domain (Si ) surrounding the cross-section of the scatterer.

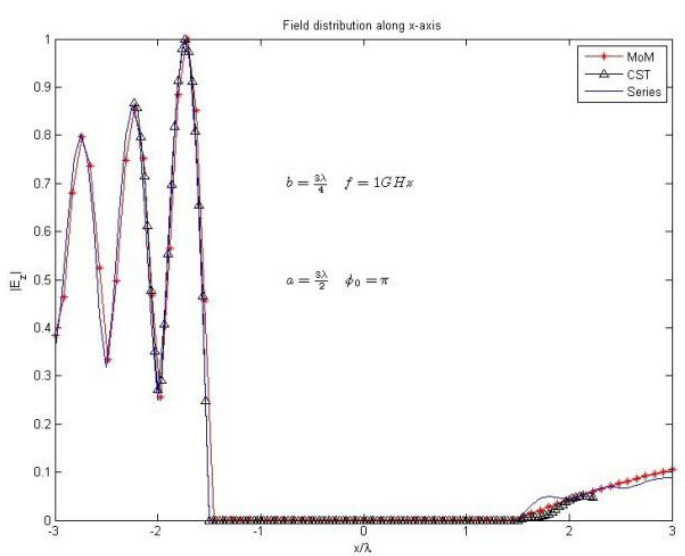

Fig. 2 Electric field distribution along the $x$-axis: the ellipse whose center is considered as the origin of $x-y$ plane has minor and major axes of $b$ and a respectively and is illuminated with a plane wave with $\varphi_{0}=180^{\circ}$ at $1 \mathrm{GHz}$ frequency $(\lambda$ denotes the wavelength in free space).

To illustrate the forward solution, an elliptical PEC cylinder has been illuminated with a plane wave impinging upon the scatterer along the $\mathrm{x}$-axis. Figure 2 shows the field distribution along the $\mathrm{x}$-axis computed with MoM and validated by its analytical solution by means of Mathieu functions. The whole scenario is also simulated in CST software to show the 
validity of the approximation replacing the $3 \mathrm{D}$ real scenario with its $2 \mathrm{D}$ counterpart under the conditions mentioned earlier. As expected and can be seen from the figure, the electric distribution within the PEC is zero and there is a good agreement among the three forward solvers.

\subsection{The Inversion Approach}

The starting point for the formulation of LSM is the following discretized Fredholm equation of the first kind:

$$
\left[E^{s}\right]_{N \times N}\left[\psi_{p}\right]_{N \times 1}=\left[g_{p}\right]_{N \times 1}(1)
$$

where $N$ denotes the number of transceivers used to illuminate and gather fields in far field, $\mathrm{E}^{\mathrm{s}}$ is the scattered electric field measured in the locations of the probes, $\Psi \mathrm{p}$ is an unknown function which can be used as the argument for an indicator function to determine the shape and location of the scatterer within the investigation domain ( $\mathrm{p} \in \mathrm{Si}$ ), and elements of the vector $g_{p}$ symbolize the far-field pattern of free-space Green function as follows:

$$
g_{p_{i}}\left(\widehat{r}_{i}, p\right)=\frac{e^{-i \pi / 4}}{\sqrt{8 \pi k_{0}}} e^{i k_{0} \hat{r}_{i} \cdot p}
$$

where $\mathrm{k}_{0}$ is the wavenumber of free-space and $\hat{r}_{i}$ stands for the unit vector pointing at the location of the $i^{\text {th }}$ probe. It is noteworthy that because of being ill-conditioned, equation (1) needs to be regulated by a regularization scheme like Tikhonov ${ }^{[5]}$. Finally, $\Psi_{\mathrm{p}}$ resulting from (1) can be used in a monotonic function like $-\log \left(\Psi_{\mathrm{p}}\right)$ to determine the border of the unknown scatterer.

\section{Constraints and Considerations of the Method}

To result in satisfactory reconstructions when implemented, LSM needs to take into account some consideration as follows. By applying LSM to some simple scenarios, these subsections try to show how implementing limitations can wreak havoc on the resultant images and deteriorate them to some extent.

\subsection{Noise Effect}

One of the important factors playing a role in real scenarios is the presence of noise. To imitate the noisy realistic environment, raw data has been corrupted by adding some white Gaussian noise. Figure 3 shows the reconstruction of the elliptical cylinder discussed in section 2.1 under both noiseless and noisy conditions. As another example, reconstruction of a circular cylinder which is a special case of the elliptical one has been investigated in Figure 4 .

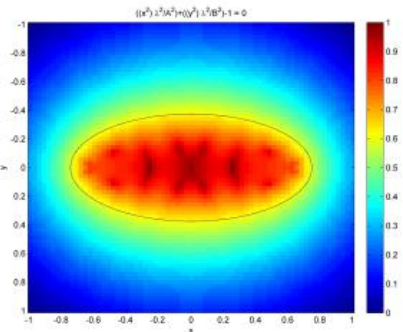

(a) Noiseless

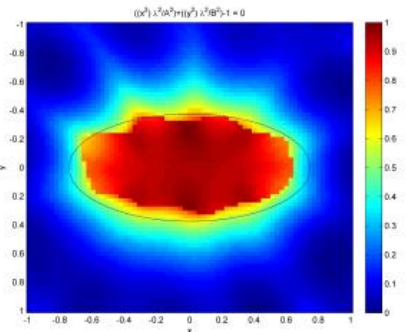

(b) $\mathrm{SNR}=20 \mathrm{~dB}$

Fig. 3 Reconstruction of an elliptical homogeneous and lossless cylinder with $\varepsilon_{\mathrm{r}}=1.8$. The solid black line shows real border of the target.

Widely used as a standard to produce binary images with a specific threshold, the fact that the maximum rate of change is more likely to occur around the border of the target (when the 2D gradient of the reconstructed image is calculated) gives us a barometer to assess the stability of the reconstructions. Although the impact of noise on the reconstructions is palpable, the algorithm can be considered robust against noise because according to Figure $4 \mathrm{c}$ the algorithm is still able 
to detect the border of the target even when the raw data is buried under heavy noise.

\subsection{Mutual Coupling Effect}

It is shown $\mathrm{in}^{[4]}$ that to have a reliable reconstruction, number of antennas $(\mathrm{N})$ and the distance between them $(\Delta)$ have to satisfy the following conditions:

$$
\begin{aligned}
& N>2 k_{0} a \\
& \Delta \cong \frac{R A}{N}
\end{aligned}
$$

where $\theta$ is the whole angular view of a scan line with equidistant elements, $\mathrm{R}$ is the radius of the circle housing elements, and considering a priori information a is the minimum radius of a circle that can enclose the estimate cross-section of the unknown target.

To illustrate the issue, the scenario shown in Figure 5 is simulated in CST where 24 monopole antennas encircle

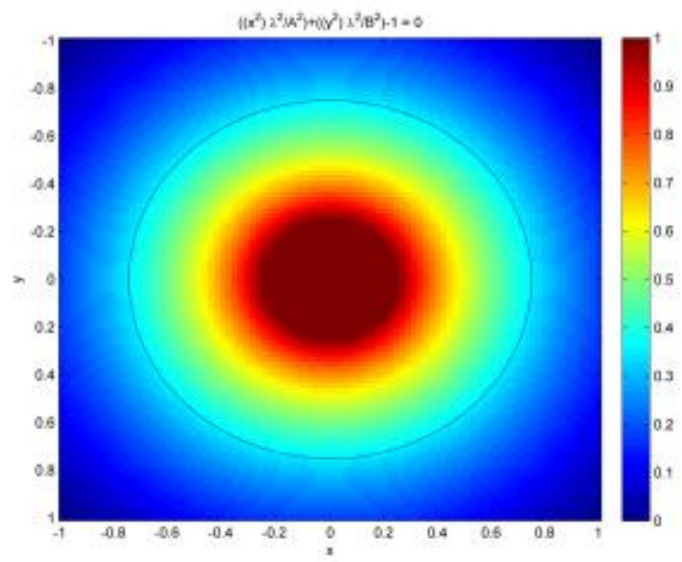

(a) Noiseless

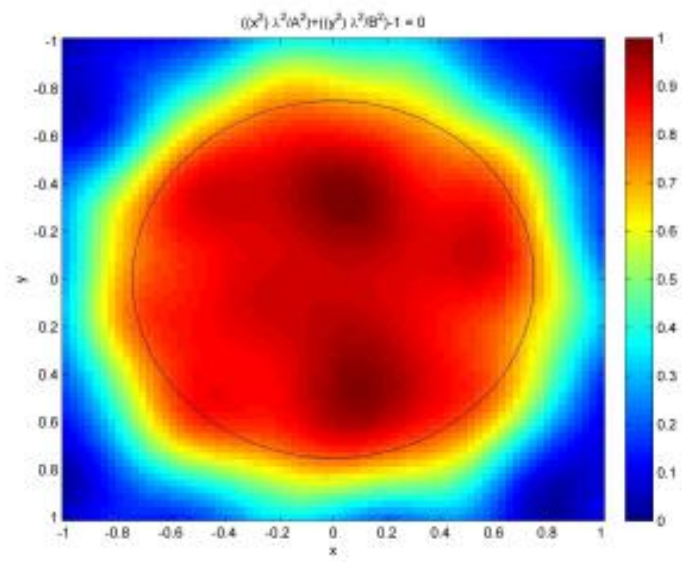

(b) $\mathrm{SNR}=5 \mathrm{~dB}$

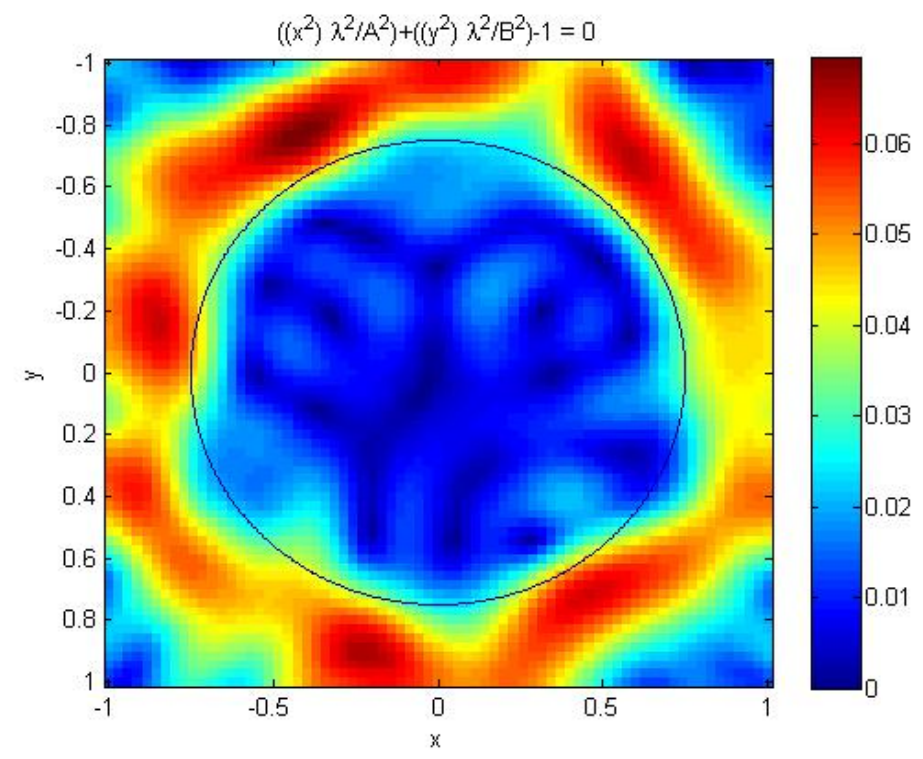

(c) Gradient of the noisy reconstruction

Fig. 4 Reconstruction of a circular counterpart of the elliptical cylinder: the solid black line shows real border of the target. a cylinder with a rectangular cross-section. Since the measurements are carried out in a multistatic modality, the mutual 
coupling effect among antennas can be significant. The working frequency, however, can be selected so that the undesirable effect of coupling is kept to a minimum ${ }^{[7]}$.

Shown in Figure 6 is the scattering parameters, $S_{11}$, of the monopole antennas used in the scenario when they radiate in free space in both the absence and the presence of other elements. A proper choice for working frequency is where the graphs meet in a single point and the point is well below $-10 \mathrm{~dB}$. The reconstructed image at frequency 5.408 $\mathrm{GHz}$ is presented in Figure 7.

\subsection{Impacts of a Limited Scan Line}

Quality of the resultant image would be adversely affected if we hire an aspect-limited scan line instead of a full-circle one.

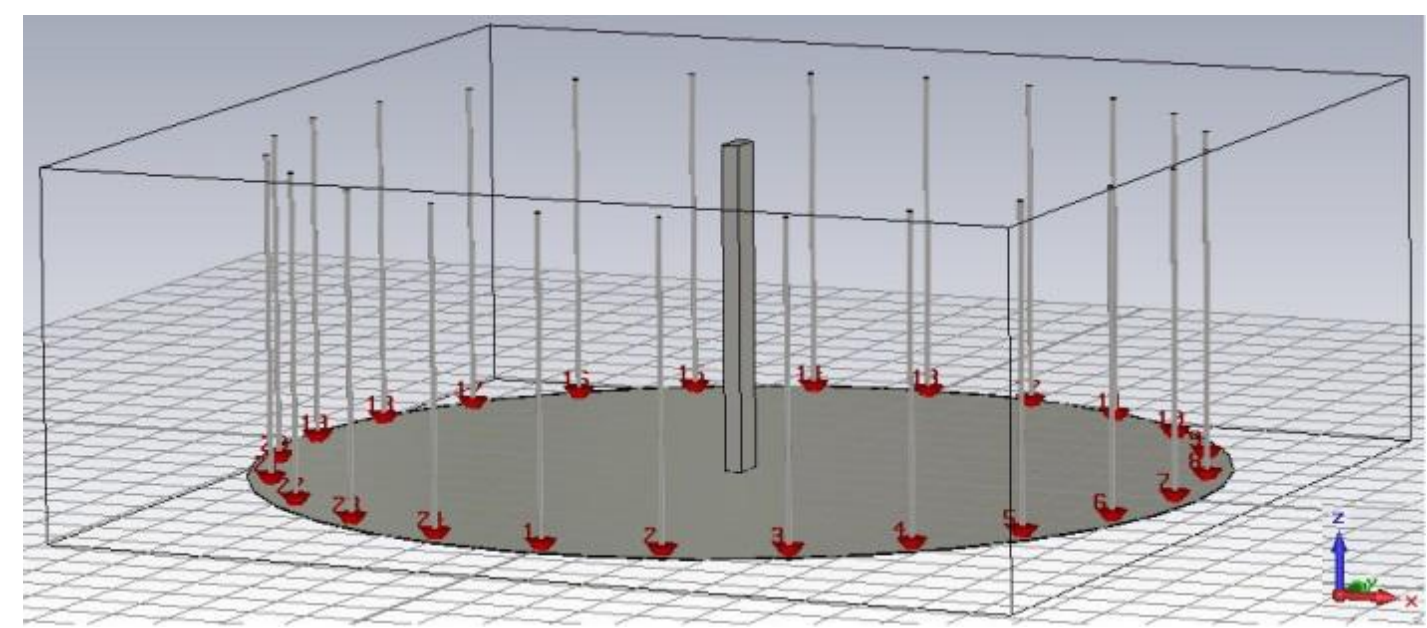

Fig. 5 The simulated scenario in CST

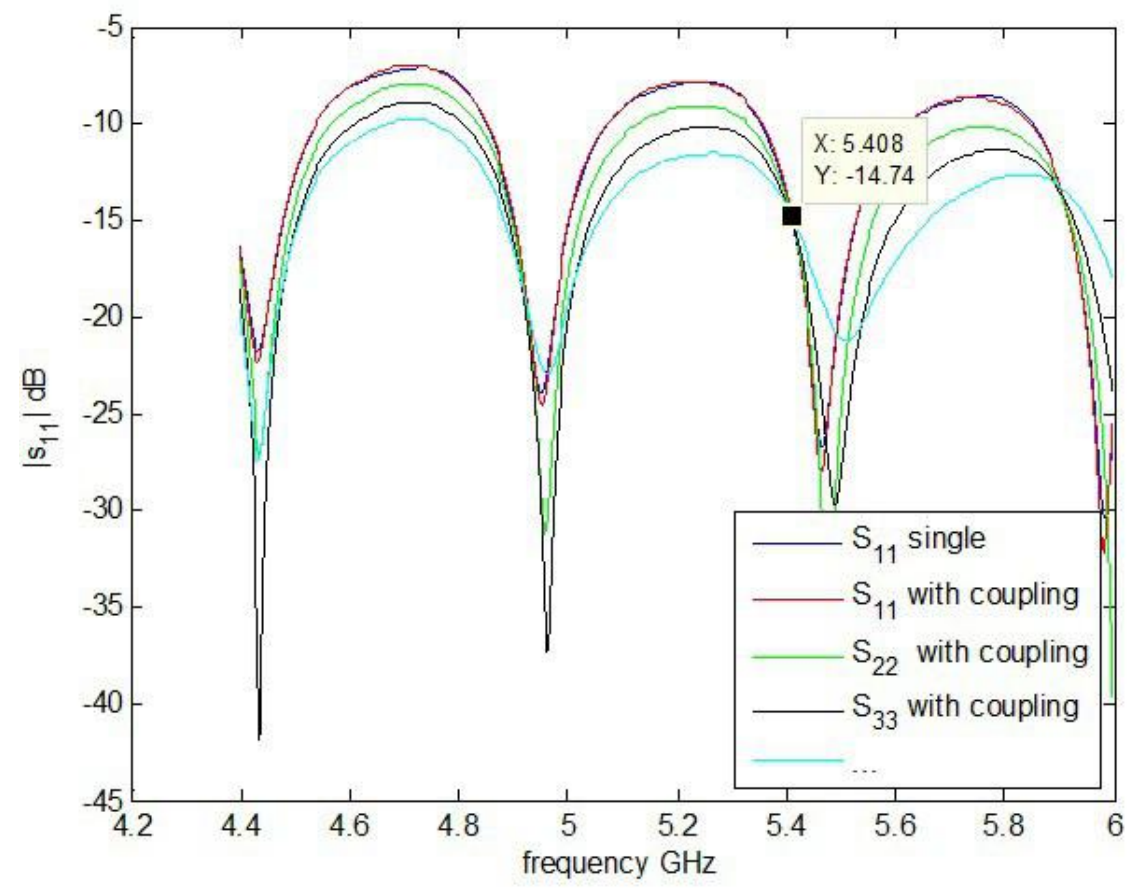

Fig. 6 Investigation of the mutual coupling effect and selection of $5.408 \mathrm{GHz}$ as a proper working frequency 


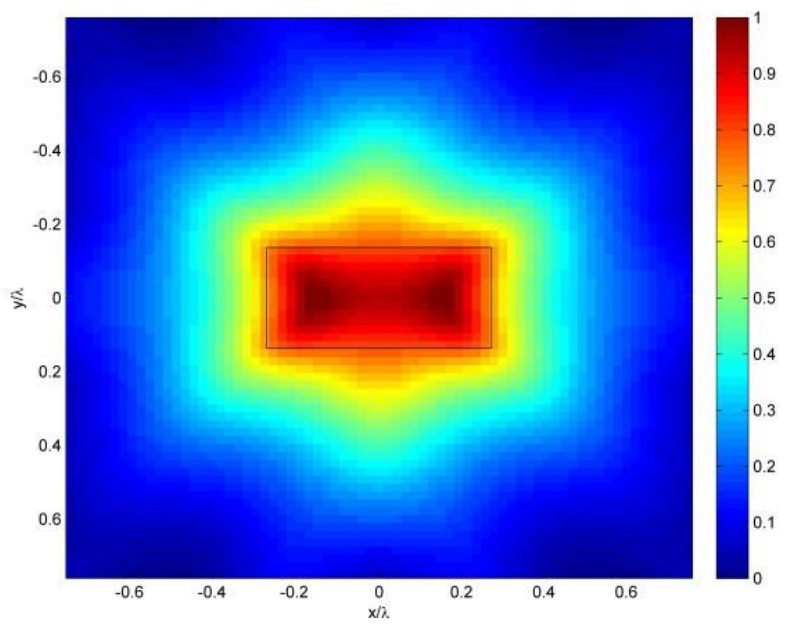

Fig. 7 Reconstruction result of the rectangular cylinder scenario. The solid black line shows real border of the target.

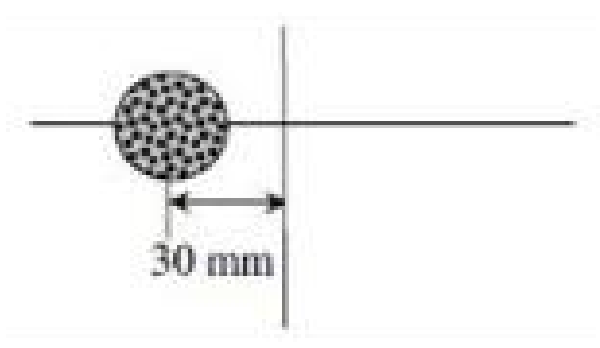

(a)

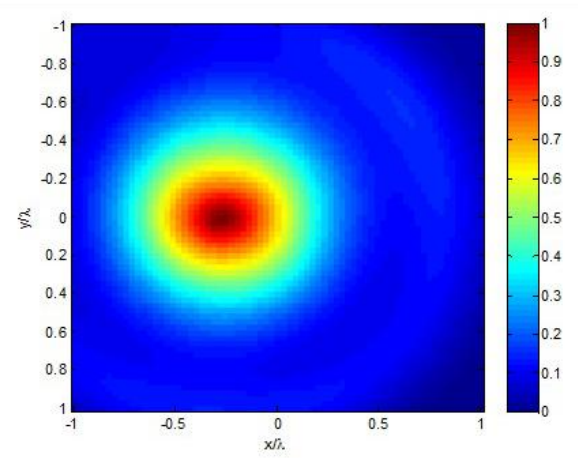

(c)

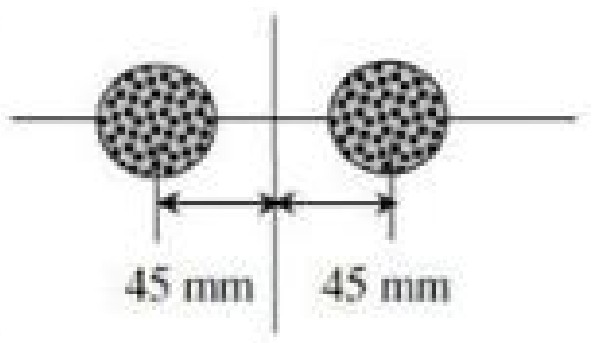

(b)

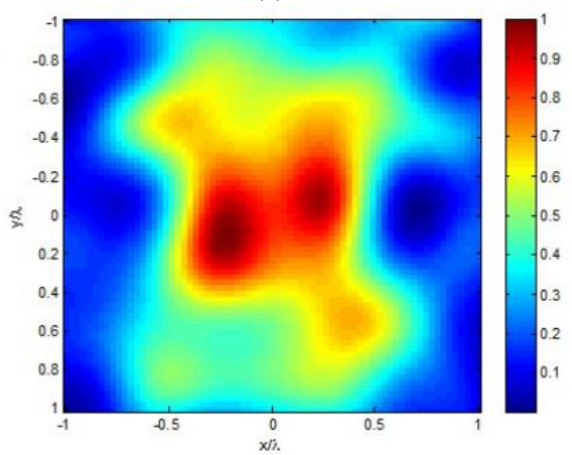

(d)

Fig. 8 Two different scenarios of Fresnel Institute's: (a) a circular dielectric cylinder, (b) two dielectric cylinders, (c) the reconstructed image of the first scenario at $2 \mathrm{GHz}$, and (d) the reconstructed image of the second scenario at the same frequency.

Frequency diversity, suggested $\mathrm{in}^{[6]}$, is a way to mitigate this effect. As a nice example of aspect-limited raw data, Fresnel Institute has provided the researchers in the field with some data sets released as an open source paper ${ }^{[8]}$. Gathered by antennas located on a sector of the circular scan line whose complete description can be read in ${ }^{[8]}$, the scattered fields are exposed to the LSM algorithm for two different scenarios depicted in Figure $8 \mathrm{a}$ and $8 \mathrm{~b}$. Since the uncovered sector of the scan line is not very big, one can still expect good results form the single frequency LSM as shown in Figures 8c and 8d.

\section{Conclusion}

Like other methods, LSM requires some a priori information such as a ballpark estimation of size of the target. This 
kind of information helps determine the minimum number of antennas as well as their inter-element space for a reliable reconstruction. Another important issue to keep in mind when implementing imaging set-ups has to with the selection of a proper frequency in which the mutual coupling of elements is the lowest. Being aspect-limited also plays a role in scenarios where it is not possible for antennas to be arranged in a full circle around the investigation domain. If not addressed properly through some techniques, sparsity in the measurement matrix can wreak havoc on the quality of the resultant images.

\section{References}

1. Colton D and Krisch A. A simple method for solving inverse scattering problems in the resonance region, Inverse Problems, 1996, 12(1): 383-393.

2. Cakoni F, Colton D and Monk P. The linear sampling method in inverse electromagnetic scattering, society for industrial and applied mathematics (SIAM), 2011, pp. 1-50.

3. Hajihashemii M. R. and El-Shenawee M. Shape reconstruction using the level-set method for microwave applications. IEEE Anten. Wireless Propag. Letter, 2008, 7: 92-96.

4. Catapano I, Crocco L and Isernia T. On simple methods for shape reconstruction of unknown scatterers. IEEE Trans. Antennas and Propagation, 2007, 55( 5) : 1431-1436.

5. Pastorino M, Microwave Imaging, 1st ed. Hoboken, New Jersey: Wiley, 2010.

6. Catapano I, Crocco L and Isernia T. Improved sampling methods for shape reconstruction of 3-D buried targets. IEEE Trans. Geoscience and Remote Sensing, 2008, 46(10):3265-3273.

7. Gilmor C, Mojabi P, Zakaria A, et al. A wideband microwave tomography system with a novel frequency selection procedure. IEEE Trans. Biomedical Engineering, 2010, 57(4): 894-904, 2010.

8. Belkebir K and Saillard M. Special section: Testing inversion algorithms against experimental data. Inverse Problems,2001, 17: $1565-1571$. 